\title{
Formation of nanostructured copper electrodes for the conversion of carbon (IV) oxide
}

\author{
Galyna Zozulia, Orest Kuntyi, Ivanna Mertsalo, Maryana Shepida, Tetyana Konda \\ Department of Chemistry and Technology of Inorganic Substances, Lviv Polytechnic National University, \\ UKRAINE, Lviv, St. Yura square, 9, E-mail: gzozula@ukr.net
}

\begin{abstract}
The basic conditions for the formation of nanostructured copper electrodes by depositing silver sols from solutions of complexes in an environment of an organic proton solvent dimethylformamide (DMF) are established. Their comparative catalytic activity for the conversion of carbon (IV) oxide has been investigated.
\end{abstract}

Keywords - galvanic replacement, nanostructured electrodes, electrolytic copper, silver, carbon (IV) oxide, conversion.

\section{Introduction}

The increasing level of carbon dioxide in the atmosphere is one of the main causes of global climate change. Among numerous strategies for its removal, storage, and reuse, electrochemical conversion of $\mathrm{CO}_{2}$ to chemicals or high value-added fuels is considered the most effective approach $[1,2]$. However, the conversion linear $\mathrm{CO}_{2}$ molecular is a kinetically slow reaction due to its low electron affinity and other energy factors. Therefore, much effort has been devoted to developing a variety of metallic catalysts. In particular, one of the effective approaches for enhancing the catalytic action is to modify metal electrocatalysts in $\mathrm{Ag}, \mathrm{Au}, \mathrm{Pd}$, and $\mathrm{Pt}$ nanostructures. By alloying of various metals, we might be able to adjust the binding strength of intermediates on the catalyst surface by means of the synergistic geometric and electronic effects between metallic components. A change in the binding strength of intermediate compounds causes a change in the reaction kinetics, as well as nature final products of $\mathrm{CO}_{2}$ conversion.

One of the promising methods for modifying the surface of metal electrodes with nanoscale particles is a galvanic replacement [3, 4]. However, the spontaneity of this process makes it difficult to obtain nanoparticles with given characteristics - geometry, size distribution, and component content. Therefore, it is relevant to establish the basic parameters that affect these characteristics.

The aim of the work was to establish the conditions for the formation of nanostructured copper electrodes for carbon (IV) oxide conversion by depositing silver precipitates in an organic aprotic solvent (DMF), which is a continuation of systematic studies on the modification of metal and semiconductor surfaces by mono- and bimetallic by galvanic replacement $[3,4]$.

\section{Research results}

It has been established that on the surface of electrolytic copper (from acidic sulfate and pyrophosphate electrolytes), nanostructured silver deposits are formed by galvanic replacement in dimethylformamide solutions. High-donor properties of dimethylformamide make the formation of surface complexes on the germs of renewable silver similar to the action of surfactants, which positively affects the nanostructure of the sediment. Unlike water, the polar molecules of DMF, due to higher electron donor properties, form a solvent $\left[\mathrm{Ag}(\mathrm{DMF})_{\mathrm{n}}\right]^{+}$soluble in Argentum, which cause cathode polarization, which also causes the formation of nanoscale spheroidal silver particles. 
It is shown that the main parameters of the influence on the morphology of silver sediment and the geometry of its structural particles are the nature, and composition of the solution, temperature and duration of the process. Micro and nanostructured silver sediments are formed in solutions of complexes of reducing metals $\left[\mathrm{Ag}(\mathrm{CN})_{2}\right]^{-},\left[\mathrm{Ag}\left(\mathrm{NH}_{3}\right)_{2}\right]^{+},\left[\mathrm{Ag}\left(\mathrm{SC}\left(\mathrm{NH}_{2}\right)_{2}\right)_{2}\right]^{+}$on copper surfaces. With increasing stability of the complex there is a tendency to decrease the size of metal particles on the substrate surface. In particular, in solutions $\left[\mathrm{Ag}(\mathrm{CN})_{2}\right]$ nanoparticles of up to $100 \mathrm{~nm}$ is formed with a relatively small range of their sizes.

As the concentration of the complex in the solution decreases, there is also a tendency to decrease the size of the particles of reducing silver, which is caused by concentration polarization. Increasing the temperature and duration of galvanic substitution contributes to the growth of particles and the formation of film sediment on the surface of electrolytic copper.

The comparative catalytic activity of the conversion of carbon (IV) oxide in $0.1 \mathrm{M} \mathrm{KHCO}_{3}$ aqueous solutions saturated with $\mathrm{CO}_{2}$ was studied on the copper cathodes obtained by modified nanostructured silver galvanic replacement. The analysis of cyclic voltammetric curves has shown that high catalysis activity is characterized by cathodes with a developed surface, obtained by electrochemical deposition of metal from acidic sulfate electrolytes. Modification of copper electrodes by nanostructured silver from cyanide complexes contributes to the deep conversion of carbon (IV) oxide.

\section{Conclusion}

1. In solutions of Argentum complexes, submicronous and nanosized silver particles with uniform distribution on the substrate surface are formed by galvanic replacement on the surface of electrolytic copper.

2. During the recovery of silver from solutions of stable cyanide complexes in a DMF medium, discrete metal particles up to $100 \mathrm{~nm}$ are formed, which facilitates the modification of the surface of copper by nanostructured sediments.

3. The increased catalytic activity of copper electrodes modified by silver from cyanide complexes is due to synergistic and nanosized effect.

\section{Acknowledgements}

This work was financially supported by the Ministry of Education and Science of Ukraine under the project "Controlled electrochemical synthesis of metal nanoparticles and nanostructured materials” (State Registration № 0118U000268).

\section{References}

[1] A.S. Reis Machado, M. Nunes da Ponte, " $\mathrm{CO}_{2}$ capture and electrochemical conversion", Green and Sustain. Chem., vol. 11, pp. 86-90, 2018.

[2] Jun-Hao Zhou, Ya-Wen Zhang, "Metal-based heterogeneous electrocatalysts for reduction of carbon dioxide and nitrogen: mechanisms, recent advances and perspective" React. Chem. Eng., vol. 3, pp. 591-625, 2018

[3] O.I. Kuntyi, G.I. Zozula, O.Ya. Dobrovets'ka, S.A. Korniy, O.V. Reshetnyak "Deposition of Copper, Silver and Nickel on Aluminum by Galvanic Replacement”, Materials Sci., vol. 53, pp. 488-493, 2018.

[4] O.I. Kuntyi, M.V.Shepida, L.Sus, G.I. Zozula, S.A. Korniy "Modification of silicon surface with silver, gold and palladium nanostructures via galvanic substitution in DMSO and DMF solutions", Chemictry \& Chemical Technology, vol. 12, pp. 305-309, 2018. 